\title{
The Role of Imaging In The Diagnosis of Pulmonary Embolism
}

\author{
Alfian Nur Rosyid ${ }^{1 *}$, M.Yamin², Arina Dery Puspitasari ${ }^{3}$ \\ ${ }^{1}$ Department of Pulmonology, Faculty of Medicine, Universitas Airlangga, Surabaya, Indonesia - Dr. Soetomo General \\ Hospital Surabaya, Indonesia \\ ${ }^{2}$ Department of Radilogy, Faculty of Medicine, Universitas Airlangga, Surabaya, Indonesia - Dr. Soetomo General Hospital \\ Surabaya, Indonesia \\ ${ }^{3}$ Department of Pharmacy, Universitas Airlangga, Surabaya, Indonesia
}

\section{AR T I C L E I N F O}

\section{Article history:}

Received 15 May 2019

Received in revised form 25 June 2019

Accepted 28 June 2019

Available online 30 June 2019

\section{Keywords:}

Acute pulmonary embolism, Imaging,

Diagnostic,

Angiography.

\section{*) Corresponding author:}

alfian-n-r-10@fk.unair.ac.id

\begin{abstract}
A B S T R A C T
Pulmonary embolism is a common condition and sometimes can be life-threatening. A proper diagnosis can reduce mortality. Some examinations are needed to diagnose pulmonary embolism, including assessing the risk factors, clinical examination, D-dimer tests, and imaging. Imaging is necessary when the previous assessment requires further investigation. There are more imaging that can be used to diagnose and assess the severity of pulmonary embolism. However, it is still controversial regarding imaging modalities for optimizing pulmonary embolism diagnose. Chest $\mathrm{X}$-Ray cannot exclude pulmonary embolism, but it is needed to guide the next examinations and to find alternative diagnoses. Pulmonary Multi-Detector CT Angiography is the gold standard to diagnose pulmonary embolism.
\end{abstract}

\section{Introduction}

Acute pulmonary embolism is a cardiovascular disease that is often fatal. Pulmonary embolism and Deep Venous Thrombosis (DVT) are part of venous thromboembolism with similar pathological processes. ${ }^{1,2}$ Pulmonary embolism occurs because dislodge of a part of the thrombus in a vein in the lower extremity or pelvis. The thrombus will follow the blood flow to the pulmonary artery and cause a blockage. This condition will increase pulmonary vascular resistance resulting in a dilatation of right ventricle following an increase of right ventricular pressure. ${ }^{3}$

The prevalence of pulmonary embolism is about $0.4 \%$, with a fatality rate of around $2-10 \%$, while the undiagnosed ones reach $5-30 \%{ }^{2}$ Acute pulmonary embolisms are the third most common cardiovascular disease after myocardial infarction and stroke. Pulmonary embolism often occurs in patients with certain risk factors including old age, a history of venous thromboembolism (pulmonary embolism or DVT), active cancer status, neurological abnormalities with paralysis, post-surgery, prolonged bed rest and congenital or acquired thrombophilia. ${ }^{1}$ Some studies have not associated other factors such as estrogen therapy, congestive heart failure, heart disease, trauma, smoking, and pregnancy as risk factors of pulmonary embolism. ${ }^{2}$ However, about one-third of pulmonary embolism cases occur without risk factors (idiopathic or non- triggered pulmonary embolism). ${ }^{1}$ As many as $60-80 \%$ cases of pulmonary embolism associated with complications of DVT. ${ }^{4}$ The diagnosis of pulmonary embolism is difficult to make, especially with the presence of heart or lung disease. False diagnosis can be fatal and deadly, but overdiagnosis results in the administration of anticoagulants that is not necessary and may harmful. ${ }^{5}$ Pulmonary embolisms are more fatal than DVT, besides having a higher recurrence and often asymptomatic. ${ }^{6}$ Fatal pulmonary embolism can be associated with hypotension, shock, and shortness of breath. ${ }^{7}$

Patients with suspected pulmonary embolism with correct examination strategies will reduce the probability of false diagnosis. ${ }^{1,2}$ The examination includes clinical assessment, risk factors, D-dimer tests, and or imaging. Imaging is needed if the previous evaluation needs further examinations. Diagnosis is applied individually according to the patient's condition. ${ }^{7}$ Diagnosis algorithm of pulmonary embolism is arranged to help assign a diagnosis. Patients without pulmonary embolism have an error rate of $<1 \%{ }^{6}$

Clinical assessments include the Well score or revised Geneva score. ${ }^{8,9}$ Imaging will be necessary after clinical evaluation. The imaging that often used is Multi Detection CT scan (MDCT) ${ }^{10,11}$ Lung CT scan (CTPA). ${ }^{11-}$ ${ }^{16} \mathrm{~V} / \mathrm{Q}$ scan (Ventilation - Scintigraphy Perfusion), ${ }^{12}$ and MRI. ${ }^{13}$ Here we review all imaging modality that 
used to optimizing diagnose and assess the severity of pulmonary embolism.

Table 1. The risk factors of vein thromboembolism

\begin{tabular}{|c|c|c|}
\hline $\begin{array}{l}\text { High risk } \\
(\mathrm{OR}: 10)\end{array}$ & $\begin{array}{c}\text { Moderate risk } \\
\text { (OR: 2-9) }\end{array}$ & $\begin{array}{c}\text { Mild risk } \\
(\mathrm{OR}<2)\end{array}$ \\
\hline $\begin{array}{l}\text { Fracture(spine/ } \\
\text { lower extremity) } \\
\text { Spine/knee } \\
\text { replacement } \\
\text { Major surgeries } \\
\text { Severe injuries } \\
\text { Spinal cord } \\
\text { injuries }\end{array}$ & $\begin{array}{l}\text { Knee arthroscopy } \\
\text { Chemotherapy } \\
\text { Heart diseases } \\
\text { Respiratory failure } \\
\text { Hormonal therapy } \\
\text { Malignancy } \\
\text { Oral contraception } \\
\text { Stroke with paral- } \\
\text { ysis } \\
\text { Pregnancy/post- } \\
\text { partum } \\
\text { History of throm- } \\
\text { boembolism } \\
\text { Thrombophilia }\end{array}$ & $\begin{array}{l}\text { Bed rest }>3 \\
\text { days } \\
\text { Immobilization } \\
>8 \text { hours } \\
\text { Older age } \\
\text { Laparoscopy } \\
\text { Obesity } \\
(\text { BMI }>40 \mathrm{~kg} / \\
\mathrm{m} 2) \\
\text { Varicose vein }\end{array}$ \\
\hline
\end{tabular}

\section{Symptoms}

Pulmonary embolism has various and unspecific symptoms, making the diagnosis is hard to make. The patients can be asymptomatic to a severe and life-threatening condition, especially in cases of acute and massive pulmonary embolism. ${ }^{7}$

The most common symptoms are shortness of breath, chest pain, tachypnea, syncope, and coughing. A study from PISAPED (The Prospective Investigative Study of Acute Pulmonary Embolism Diagnosis) stated that 96\% of patients with pulmonary embolism complained of sudden shortness of breath, chest pain or fainting (one or a combination). ${ }^{2}$ Less common symptoms are fever, bloody coughing, cyanosis, hypotension, and shock. ${ }^{6}$ The severity is affected by the location of emboli, whether in the segment or subsegment of the pulmonary artery branches. Severe symptoms will be experienced by the patient with massive emboli. ${ }^{2}$ Pulmonary embolism can be accompanied by DVT; if both present, the sign can be swollen, painful, warm, and red extremity.

Patients presenting shortness of breath associated with heart and lung or other diseases may show more severe symptom and can be an indication of pulmonary embolism. ${ }^{6}$ The presence of comorbid factors increases the difficulty of diagnosis making. ${ }^{1}$

Table 2. Well score for diagnosing pulmonary embolism

\begin{tabular}{lc}
\hline \multicolumn{1}{c}{ Criteria } & Score \\
\hline $\begin{array}{l}\text { Sign and symptoms of DVT (swol- } \\
\text { len, painful, extremity) }\end{array}$ & 3 \\
\hline $\begin{array}{l}\text { Suspicion of pulmonary embolism is } \\
\text { higher than another diagnosis }\end{array}$ & 3 \\
\hline Heart rate $>100$ bpm & 1.5 \\
\hline $\begin{array}{l}\text { Immobilization or history of surgery } \\
\text { in the past four weeks }\end{array}$ & 1.5 \\
\hline History of DVT or PE & 1.5 \\
\hline Bloody cough & 1 \\
\hline Malignancy (on therapy or palliative) & 1 \\
\hline Note: Score $<2:$ low probability (3.6\%) \\
$\begin{array}{l}\text { Score 2-6: moderate probability }(20.5 \%) \\
\text { Score }>6: \text { high probability }(66.7 \%)\end{array}$
\end{tabular}

Table 3. Revised Geneva score to diagnose pulmonary embolism

\begin{tabular}{lc}
\hline \multicolumn{1}{c}{ Criteria } & Score \\
\hline Age $>65$ years & 1 \\
\hline History of DVT or PE & 3 \\
\hline $\begin{array}{l}\text { Surgery (general anesthesia) or lower extremity } \\
\text { fracture in the past four weeks }\end{array}$ & 2 \\
\hline Malignancy & 2 \\
\hline Pain in unilateral lower extremity & 3 \\
\hline Bloody cough & 2 \\
\hline Heart rate $75-94$ bpm & 3 \\
\hline Heart rate $\geq 95$ bpm & 5 \\
\hline $\begin{array}{l}\text { Lower extremity tenderness or unilateral } \\
\text { edema }\end{array}$ & 4 \\
\hline
\end{tabular}

\section{Diagnosis of Pulmonary Embolism}

An objective assessment to diagnose pulmonary embolism is needed because clinical evaluation alone is not reliable. There is no single examination that has ideal properties (sensitivity and specificity values $100 \%$, no risk, and low cost) $[1,6]$. Diagnosis of pulmonary embolism requires several tests to rule out the differential diagnosis and establish the diagnosis. Some collegial such as the American College of Physicians, American Academy of Family Physicians, British Thoracic Society, and European Society of Cardiology have made algorithms to ease the diagnosis making $[5,6]$.

Patients with suspected pulmonary embolism must undergo risk factor assessment and physical examination. Physical examination findings can vary, such as tachypnea, tachycardia, hypoxia, fever, cyanosis, and increased JVP[2]. Blood Gas Analysis, chest X-ray and ECG (Electrocardiography) are needed to rule out a differential diagnosis, but the results are often insensitive and not specific[2]. Cardiac biomarkers (Troponin T and I) and echocardiography can be used to assess the severity of pulmonary emboli related to prognosis.[5]

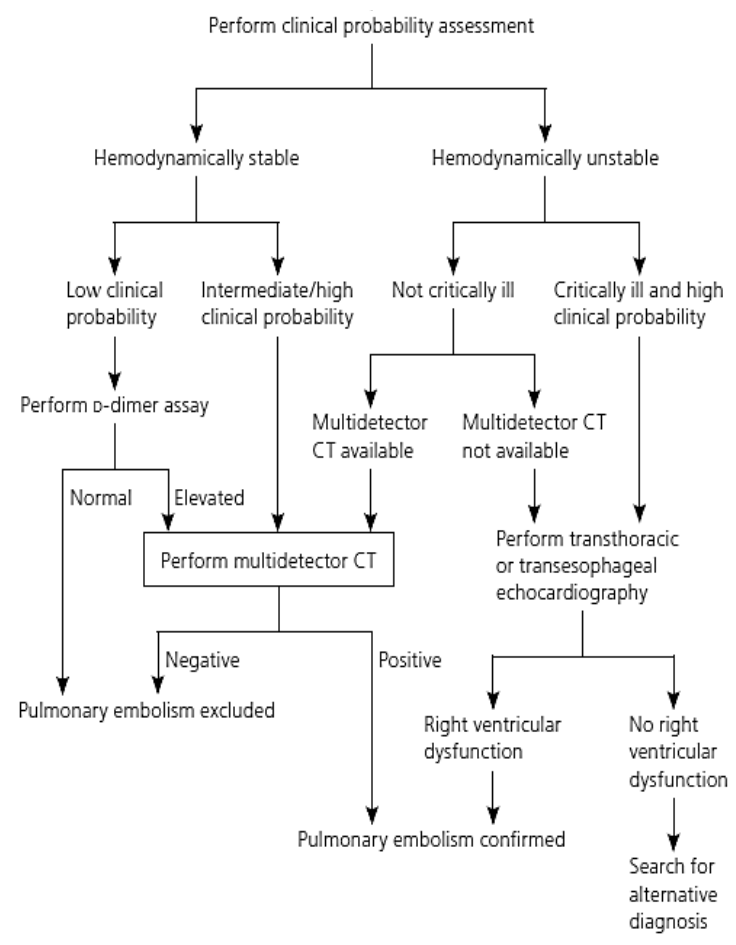

Figure 1. Diagnosis algorithm of pulmonary embolism ${ }^{6}$ 


\section{a. Blood Gas Analysis}

From Blood Gas Analysis obtained hypoxemia, hypercapnia, and an increase in AaDO2. However, normal Blood Gas Analysis can be found in the young patient. ${ }^{5}$

\section{b. D-dimer examination}

The d-dimer examination is mandatory to accompany clinical assessment. A negative result in patients with low probability indicates no need for further imaging. While the D-dimer test is not needed in high-risk patients. ${ }^{5}$

Plasma D-dimer has degraded fibrin through fibrinolysis. ${ }^{8}$ This examination is a test with high NPV (Negative Predictive Value) and low PPV (Positive Predictive Value). This examination is the first choice in patients with low to moderate probability. The D-Dimer concentration $>0.5 \mathrm{mg} / \mathrm{L}$ has a sensitivity of $95 \%$ and a specificity of $55 \%$ to diagnose VTE (Venous Thromboembolism). ${ }^{1}$

\section{c. Cardiac Marker Examination}

Troponin $\mathrm{T}$ and I examination is used to assess myocardial infarction but can be increased in patients with severe pulmonary embolism. So that this examination cannot be used to rule out a diagnosis of pulmonary embolism. While an increase in BNP (Brain Natriuretic Peptide) is related to right ventricle dysfunction due to pulmonary embolism. ${ }^{5}$ BNP can only be used as a biomarker in low-risk pulmonary embolism. ${ }^{3}$

\section{d. Electrocardiography (ECG)}

ECG results can be sinus tachycardia, specific T-wave changes, p-pulmonale, right ventricular strains, Right Bundle Branch Blocks (RBBB), S1, Q3, T3 (S waves in the lead I, Q waves in lead II and waves T inversion in the lead III). ECG is useful to rule out the differential diagnoses such as myocardial infarction. ${ }^{5}$

\section{Imaging of Pulmonary Embolism a. Chest X-Ray}

PA (posteroanterior) and lateral Chest X-Ray are important in evaluating pulmonary embolism. Patients with chest pain showing pneumonia infiltrates, massive pleural effusion, pneumothorax, pulmonary edema14 need no additional radiological examinations. Normal chest radiograph does not rule out the diagnosis of pulmonary embolism. There is no typical picture for pulmonary embolism. ${ }^{4}$

The result of Chest X-Ray can be normal (14\%) or abnormal. Normal results in patients with hypoxia without bronchospasm support the presence of pulmonary embolism. Abnormalities found include plate atelectasis $(68 \%)$, pleural effusion (48\%), Hampton hum (35\% - opacities resembling effusions indicating distal parenchymal infarction from thrombus), increased hemidiaphragm (24\%), Fleischner's sign (15\% - prominent central pulmonary arteries), Westermark's sign (7\% - peripheral oligemia), cardiomegaly (7\%) and pulmonary edema $(5 \%)$. Other abnormalities are rarely found in pulmonary embolism. ${ }^{5}$

\section{b. Echocardiography}

A transthoracic or transesophageal echocardiogram is not indicated to diagnose acute pulmonary embolism. ${ }^{4}$ Echocardiography is essential to assess right ventricular dysfunction in patients with pulmonary embolism, due to the prognosis and mortality in pulmonary embolism and thromboembolism. ${ }^{10}$ Right Ventricular dysfunction has some signs, they are right ventricular dilatation, hypokinetic wall, opposite septal wall movement, pulmonary arterial dilation, right - left ventricular systolic pressure gradient $>30 \mathrm{mmHg}$ and acceleration time of pulmonary artery rate $<80$ milliseconds. ${ }^{3}$

Diagnosis of right ventricular dysfunction can be made if two of the following findings are founded, namely RV / LV end-diastolic diameter ratio $>0.9$ (apical four chambers) or RV / LV end-diastolic $>0.7$ (parasternal long axis or substernal fourchamber) or opposite movement of interventricular septum or systemic-pulmonary arterial pressure $>30 \mathrm{mmHg}[10]$. The presence of right ventricular dilatation is more happens in the main pulmonary artery than in the segmental or subsegmental embolism. ${ }^{14}$

\section{c. Ultrasound}

Extremities venous ultrasound can be indicated if suspected since there is a correlation between pulmonary embolism and DVT. The examination used is duplex Doppler ultrasound with leg compression or continuous-wave Doppler. The presence of DVT does not necessarily indicate the presence of pulmonary embolism, but increase the suspicion of pulmonary embolism. Normal ultrasound results also do not exclude pulmonary embolism but reduce the suspicion. ${ }^{4}$

\section{d. CT Pulmonary Angiography (CTPA)}

CTPA has an essential role in pulmonary embolism diagnosing since 1992. Technological advances in CT - from helices to multidetector increases the resolution in pulmonary artery imaging, either large or small. CTPA is more sensitive and specific than conventional angiography, especially at the subsegmental level. Differences in CTPA interpretation have proven to be better for segmental levels than V / Q scan[4]. MDCT (Multidetector Computed Tomography) has a sensitivity of $83-100 \%$ and specificity $89-98 \%$ [15]. Pulmonary angiography is the gold standard to diagnose pulmonary embolism. ${ }^{3}$

The accuracy of CTPA is higher when combined with clinical assessment and D-dimer test. Positive CTPA results in patients with high or moderate suspicion; the positive predictive value is also high. If the CTPA is negative at low clinical suspicion, the diagnosis of pulmonary embolism can be excluded. ${ }^{4}$

CTPA has fewer "nondiagnostic" findings than V/Q scan. The CTPA false negative level is deficient. The results of the study showed no adverse effects on patients with negative CTPA and were not treated. The combination of multidetector CTPA and D-dimer test has very high positive and negative predictive values. Also, CTPA sometimes shows pathology other than pulmonary embolism associated with patient symptoms. ${ }^{4}$

Besides being useful in direct imaging of the presence of thrombus in the pulmonary artery, CT scan can also classify the risk of pulmonary embolism by measuring the diameter of the right/left ventricle (RV / LV). This result is useful to determine prognosis and treatment. ${ }^{4}$ It is stated that MDCT is more accurate in assessing right Ventricular dysfunction than Echocardiography, by measuring the ratio of RV / LV. ${ }^{10}$

Axial CT scan with an RV / LV ratio of 0.9-1.5 indicates right ventricular dysfunction with poor prognosis. This ratio has a low positive predictive value, but if the rate is $<1$, it will have a high negative predictive value.

The previous study showed that there was a relationship between mortality of pulmonary embolism and the diameter of the Azygos vein, but the correlation is negative in the dilation of Superior and Inferior Vena Cava. It was also stated that there 
was no correlation between Aorta Ascending diameter and mortality of pulmonary embolism. ${ }^{3,14}$ Diagnosis of pulmonary embolism can be made when there is partial or total pulmonary artery defect filling at least in two consecutive images and located in the center of a blood vessel, or has a sharp angle to the vessel wall. The location is evaluated at the level of the involved artery and related lobar. Location then categorized as central (for example, main pulmonary artery, central pulmonary artery, and both interlobar pulmonary artery), lobar, segment, and subsegment. ${ }^{11,15}$ Lobar pulmonary embolism is classified based on anatomy: upper right lobe, lobe right middle, lower right lobe, upper left lobe, ligula, and left lower lobe. Location and level of PE are determined based on the number of embolisms because some patients can have more than one embolism. ${ }^{11}$

Some studies linked the clot index to the mortality of pulmonary embolism, but that was considered insignificant. A recent study stated that clots in the center of the pulmonary artery could be a predictor of mortality. Signs of right ventricular dysfunction (RV / LV ratio > 1) and obstruction index $>40 \%$ showed an increased mortality within 90 days (PPV 18.8). Another study stated that the RV / LV >1.5 ratios with the obstruction index $>50 \%$ showed severe pulmonary embolism with poor outcomes. ${ }^{14}$ The degree of obstruction is divided into three; they are without obstruction, partial obstruction and total obstruction. ${ }^{16}$
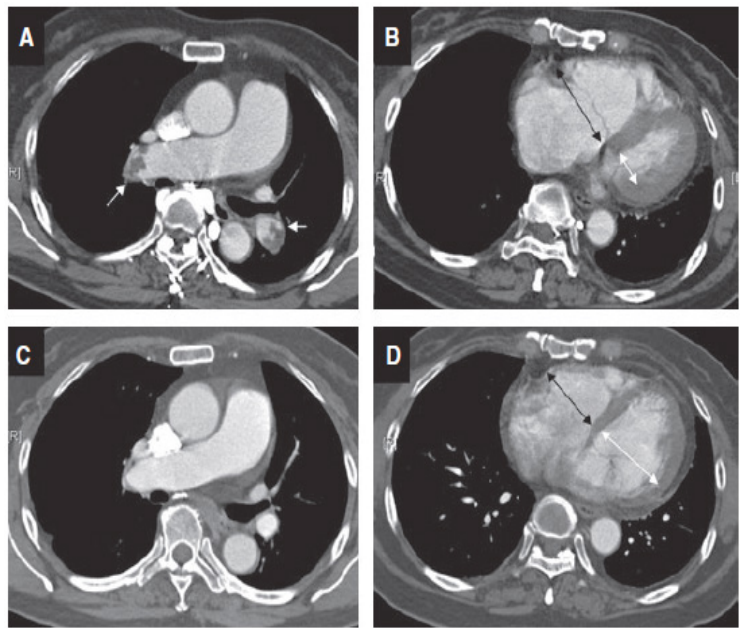

Figure 2. CTPA of patients with acute pulmonary embolism (A) Appearing embolism in the right main pulmonary artery with index clot 50\% (B) ratio RV / LV> 2 supports the presence of right ventricular dysfunction. Patients are given thrombolytic therapy, and there was an improvement (C) thrombus resolution and (D) RV / LV ratio returned to normal $(0.8) .{ }^{14}$

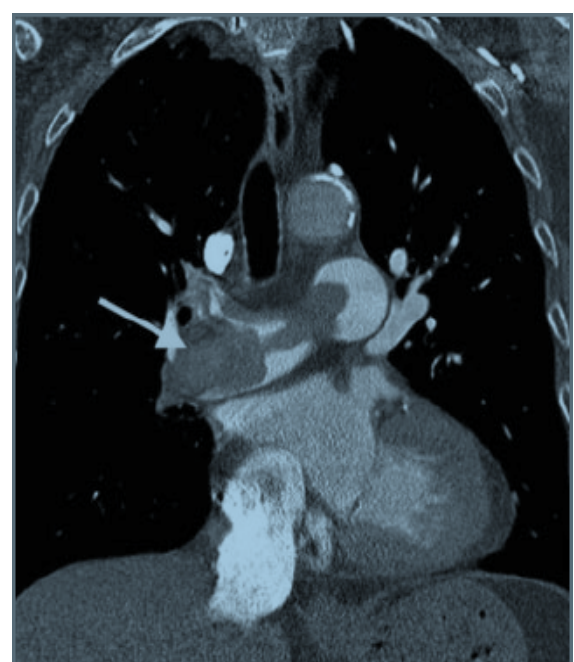

Figure 3. CTPA with central artery embolism ${ }^{13}$

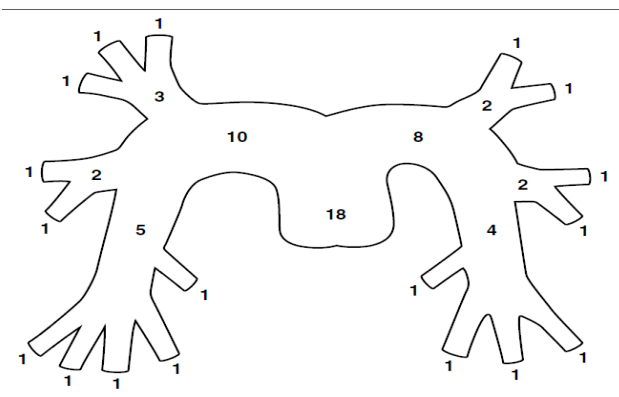

Figure 4. Schematic algorithm of pulmonary embolism. Non-occlusive segmental pulmonary artery embolism is given a score of 1 , whereas a more proximal artery was given a higher score ${ }^{17}$

An ECG guided MDCT examination will provide better accuracy in assessing the heart. However, this examination requires a longer time so that radiation exposure is also higher, so it is not used as a routine examination in an emergency. However, MDCT cannot be used to assess right ventricular function (e.g., hypokinesia or assess pulmonary arterial pressure). ${ }^{10}$ Recent technological advances such as ECG-gated and dual source CT give an accurate evaluation of pulmonary arteries, thoracic aorta, and coronary arteries. In general, multidetector CTPA is more precise than singleslice CT or V / Q scan. ${ }^{4}$

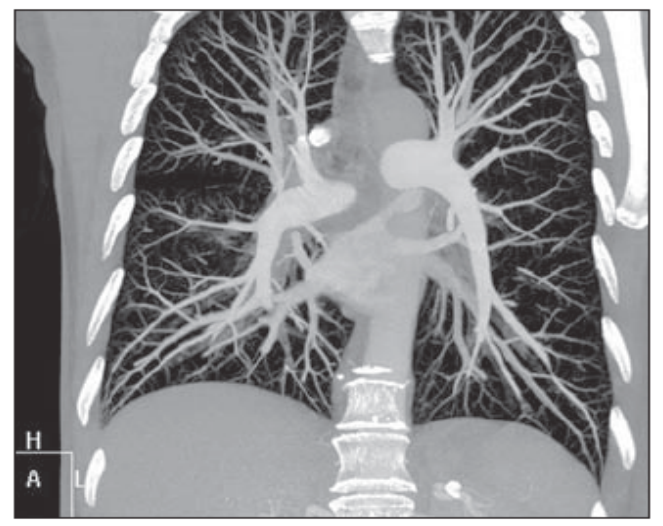

Figure 5. CT Pulmonary angiography shows central, segmental and subsegmental of pulmonary artery branches ${ }^{15}$ 
Another imaging is Perfusion Blood Volume (PBV), which is a dual-energy CT. This imaging assesses pulmonary perfusion; if there is obstruction due to thrombus, the perfusion will be disturbed on the degree of obstruction. ${ }^{16}$

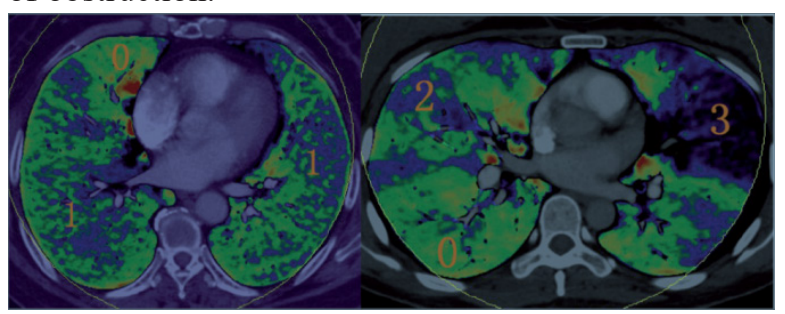

Figure 6. Classification of pulmonary perfusion defect score. Green-Red: normal perfusion, Blue-Green with even distribution: mild perfusion defect, Blue with diffuse configuration: moderate perfusion defect, blueblack: without perfusion ${ }^{16}$

Exposure to excessive ionizing MDCT radiation can increase the risk of cancer and increase mortality, but in the case of pulmonary embolism, it becomes dilemmatic because undiagnosed pulmonary embolism can be life-threatening. Therefore, the benefits and risks of radiation must be considered. The effective dose of MDCT radiation is around $9 \mathrm{mSv}$, and for women, it can be lower $(7.5 \mathrm{mSv}){ }^{18}$ Some factors that may affect the MDCT results include low sharp contrast, too many movements, and technical factors (contrast media selection, slow bolus time). ${ }^{15}$

\section{e. MRI (Magnetic Resonance Imaging)}

The use of MRI increases the accuracy of pulmonary embolism diagnosis when combined with angiography and pulmonary perfusion examination. MRI accuracy is comparable to 16 -slices MDCT. ${ }^{13}$ The advantage of MRI is radiation-free (non-ionizing) ${ }^{18}$ and can be performed without contrast so that it is safer for patients with kidney disorders and pregnant women. MRI takes time for about 10 minutes. The findings of pulmonary embolism in MRI are the same as CT scans but are divided into vascular signs and parenchymal signs. The vascular sign is in the form of a decrease in blood vessel diameter, loss of contrast under a blood vessel that is blocked by embolism. The parenchymal signs are pleural-based opacity, peripheral nodules, linear irregular opacity in the periphery, mosaic image. ${ }^{13}$

The sensitivity of the MRA is $77-100 \%$, and the specificity is $95-98 \%$. If the location of emboli in the central and lobar then the sensitivity is $100 \%$, whereas if in the segment is $84 \%$ and $40 \%$ if it is subsegment. PIOPED III suggests that the MRA is only used if there are contraindications to other examinations. ${ }^{13}$

MRA (Magnetic Resonance Angiography) and MR perfusion imaging can quickly evaluate central and segmental pulmonary arteries. MR perfusion has a high sensitivity for diagnosis and is often used in combination with MRI and MRA. MRI without MRA is not indicated as a routine examination of suspicion of pulmonary embolism because more invasive[4].

\begin{tabular}{lc}
\multicolumn{1}{c}{$\begin{array}{c}\text { Table } 4 . \text { Severity index of pulmonary embolism }[3] \\
\text { Age }\end{array}$} & $\begin{array}{c}\text { Score } \\
\text { In years }\end{array}$ \\
\hline Male & 10 \\
Malignancy & 30 \\
Heart failure & 10 \\
COPD & 10 \\
Heart rate $\geq 110 x$ bpm $\quad$ pressure & 20 \\
Systolic blood & 30 \\
$<100$ mmHg & \\
Respiratory rate $\geq 30 \mathrm{x}$ bpm & 20 \\
Temperature $<360 \mathrm{C}$ & 20 \\
Mental status alteration & 60 \\
O2 saturation $<90 \%$ & 20 \\
\hline
\end{tabular}

\section{Conclution}

A proper diagnosis of pulmonary embolism can reduce mortality. Some examinations are needed to diagnose pulmonary embolism, including assessing the risk factors, clinical examination, D-dimer tests, and imaging. Many imaging can be used to diagnose and evaluate the severity of pulmonary embolism. Although chest X-Ray cannot exclude pulmonary embolism, it is needed to guide the next examinations and to find alternative diagnoses. Pulmonary Multi-Detector CT Angiography is the gold standard to diagnose pulmonary embolism.

\section{Conflict of Interest}

The author stated there is no conflict of interest

\section{References}

1. Sadigh, G., A.M. Kelly, and P. Cronin, Challenges, Controversies, and Hot Topics in Pulmonary Embolism Imaging. AJR Am J Roentgenol, 2011. 196(3): p. 497-515.

2. Gandara, E. and P.S. Wells, Diagnosis: Use of Clinical Probability Algorithms. Clin Chest Med, 2010. 31(4): p. 629-39.

3. Abrahams-van Doorn, P.J. and I.J. Hartmann, Cardiothoracic CT: One Stop Shop Procedure? Impact on the Management of Acute Pulmonary Embolism. Insights Imaging, 2011. 2(6): p. 705-15.

4. Bettmann, M.A., et al., ACR Appropriateness Criteria(R) Acute Chestpain Suspected Pulmonary Embolism. J Thorac Imaging, 2012. 27(2): p. W28-31.

5. Hamad, M.M.A., et al., Diagnostic Approach to Pulmonary Embolism and Lessons from a Busy Acute Assessment Unit in the UK. Breathe, 2011. 7(4): p. 315-323.

6. Wilbur, J. and B. Shian, Diagnosis of Deep Venous Thrombosis and Pulmonary Embolism. Am Fam Physician, 2012. 86(10): p. 913-9.

7. Agnelli, G. and C. Becattini, Acute Pulmonary Embolism. N Engl J Med, 2010. 363(3): p. 266-74.

8. Warren, D.J. and S. Matthews, Pulmonary Embolism: Investigation of the Clinically Assessed Intermediate Risk Subgroup. Br J Radiol, 2012. 85(1009): p. 37-43.

9. Penaloza, A., et al., Comparison of the Unstructured Clinician Gestalt, the Wells Score, and the Revised Geneva Score to Estimate Pretest Probability for Suspected Pulmonary Embolism. Ann Emerg Med, 2013. 62(2): p. 117-124 e2.

10. Becattini, C., et al., Multidetector Computed Tomography for Acute Pulmonary Embolism: Diagnosis and Risk Stratification in a Single test. Eur Heart J, 2011. 32(13): p. 1657-63.

11. Lee, E.Y., et al., Pulmonary Embolism Detected by Pulmonary MDCT Angiography in Older Children and Young Adults: Risk Factor Assessment. AJR Am J Roentgenol, 2012. 198(6): p. 1431-7.

12. Sheh, S.H., et al., Pulmonary Embolism Diagnosis and Mortality with Pulmonary CT Angiography Versus Ventilation Perfusion Scintigraphy: Evidence of Overdiagnosis with CT? AJR Am J Roentgenol, 2012. 198(6): p. 1340-5. 
13. Hochhegger, B., et al., Magnetic Resonance Imaging Findings in Acute Pulmonary Embolism. Br J Radiol, 2011. 84(999): p. 2827.

14. Venkatesh, S.K., CT Pulmonary Angiography for Pulmonary Embolism: Role Beyond Diagnosis? Int J Clin Pract Suppl, 2011(171): p. iii-v.

15. Bogot, N.R., et al., Image Quality of Low Energy Pulmonary CT Angiography: Comparison with Standard CT. AJR Am J Roentgenol, 2011. 197(2): p. W273-8.

16. Zhou, Y., et al., Assessment of Correlation Between CT
Angiographic Clot Load Score, Pulmonary Perfusion Defect Score and Global Right Ventricular Function with Dual Source CT for Acute Pulmonary embolism. Br J Radiol, 2012. 85(1015): p. 972-9.

17. Morris, M.F., et al., CT Findings and Long Term Mortality After Pulmonary Embolism. AJR Am J Roentgenol, 2012. 198(6): p. 1346-52.

18. Woo, J.K., et al., Risk-benefit Analysis of Pulmonary CT Angiography in Patients with Suspected Pulmonary Embolus. AJR Am J Roentgenol, 2012. 198(6): p. 1332-9. 